\title{
Alterstice
}

Revue internationale de la recherche interculturelle

International Journal of Intercultural Research

Revista International de la Investigacion Intercultural

\section{Une brèche dans la culture, d'un bord à l'autre. Témoigner et recevoir le témoignage de la disparition forcée}

\section{Alice Verstraeten}

Volume 1, numéro 2, 2011

Droits humains et témoignages : l'épreuve de la culture

URI : https://id.erudit.org/iderudit/1077604ar

DOI : https://doi.org/10.7202/1077604ar

Aller au sommaire du numéro

Éditeur(s)

Alterstice

ISSN

1923-919X (numérique)

Découvrir la revue

Citer cet article

Verstraeten, A. (2011). Une brèche dans la culture, d'un bord à l'autre.

Témoigner et recevoir le témoignage de la disparition forcée. Alterstice, 1(2), 69-78. https://doi.org/10.7202/1077604ar

\section{Résumé de l'article}

À l'origine des " Mères de la Place de Mai » et de leur réseau militant se trouve la disparition forcée : une indicible rupture de tous les tabous. Les crimes d'enlèvement, torture et assassinat, puis l'acharnement à faire disparaître les corps sont occultés par le déni, le mensonge et l'impunité.

Les « Mères de la Place de Mai » sont néanmoins parvenues à résister : elles ont propulsé leurs témoignages dans l'espace public, elles ont rendu visibles les crimes contre l'intimité pour parvenir à dénoncer le crime contre l'humanité. Le témoignage se trouve au coeur de leur résistance, et il doit composer avec la violence. Se taire est moralement et psychiquement impossible, mais dire est impossible aussi. Les témoins apparaissent transis d'impuissance ainsi que d'un fort sentiment d'illégitimité (les vraies victimes - témoins les plus complets - étant mortes).

Le témoignage, mis en réseau, apparaît comme une réinitialisation du lien social. Mais il continue de souffrir de la défaillance de ses interlocuteurs. Personnalités clivées, stigmates de la mort du social, les témoins, en nous parlant de l'horreur, la font advenir dans nos consciences. Le témoignage est alors empêché non seulement par les bourreaux, mais aussi par la foule de ceux qui préfèrent ne pas voir et ne pas savoir. Il bute contre l'indicible et l'irreprésentable.

Entendre un témoignage de la disparition, c'est se laisser heurter par elle, c'est être ni bourreau ni neutre. C'est être empathique. C'est ainsi que, anthropologue, j'ai abordé la Place de Mai. Alors seulement, par un partage du sens, émerge la possibilité d'une culture commune entre les témoins et ceux qui les écoutent. Le témoignage est ici compris comme transculturel au sens où il ne peut naître qu'au coeur d'une médiation. Les uns remontent de la violence extrême décivilisatrice et indicible vers la parole, les autres marquent une inflexion depuis la culture vers la réification des mots, l'aporie, l'asphyxie (depuis l'endroit du décor vers son envers qui lui est indissociable). 


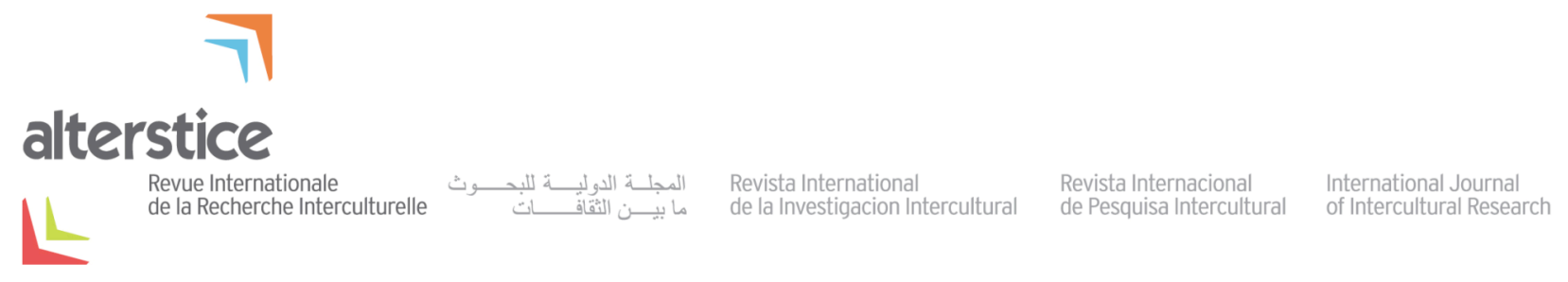

ARTICLE THÉMATIQUE

\title{
Une brèche dans la culture, d'un bord à l'autre. Témoigner et recevoir le témoignage de la disparition forcée
}

\author{
Alice Verstraeten ${ }^{1}$
}

\section{Résumé}

À l'origine des «Mères de la Place de Mai » et de leur réseau militant se trouve la disparition forcée : une indicible rupture de tous les tabous. Les crimes d'enlèvement, torture et assassinat, puis l'acharnement à faire disparaître les corps sont occultés par le déni, le mensonge et l'impunité.

Les «Mères de la Place de Mai » sont néanmoins parvenues à résister : elles ont propulsé leurs témoignages dans l'espace public, elles ont rendu visibles les crimes contre l'intimité pour parvenir à dénoncer le crime contre I'humanité. Le témoignage se trouve au cœur de leur résistance, et il doit composer avec la violence. Se taire est moralement et psychiquement impossible, mais dire est impossible aussi. Les témoins apparaissent transis d'impuissance ainsi que d'un fort sentiment d'illégitimité (les vraies victimes - témoins les plus complets - étant mortes).

Le témoignage, mis en réseau, apparaît comme une réinitialisation du lien social. Mais il continue de souffrir de la défaillance de ses interlocuteurs. Personnalités clivées, stigmates de la mort du social, les témoins, en nous parlant de l'horreur, la font advenir dans nos consciences. Le témoignage est alors empêché non seulement par les bourreaux, mais aussi par la foule de ceux qui préfèrent ne pas voir et ne pas savoir. II bute contre l'indicible et l'irreprésentable.

Entendre un témoignage de la disparition, c'est se laisser heurter par elle, c'est être ni bourreau ni neutre. C'est être empathique. C'est ainsi que, anthropologue, j'ai abordé la Place de Mai. Alors seulement, par un partage du sens, émerge la possibilité d'une culture commune entre les témoins et ceux qui les écoutent. Le témoignage est ici compris comme transculturel au sens où il ne peut naître qu'au cœur d'une médiation. Les uns remontent de la violence extrême décivilisatrice et indicible vers la parole, les autres marquent une inflexion depuis la culture vers la réification des mots, l'aporie, l'asphyxie (depuis l'endroit du décor vers son envers qui lui est indissociable).

\section{Rattachement de l'auteure \\ ${ }^{1}$ Centre de recherches et d'études anthropologiques, Université de Lyon II, Lyon, France}

\section{Correspondance}

versalice@yahoo.fr

\section{Mots clés}

témoignage; Argentine; disparition forcée; décivilisation; indicible; transmission

\section{Pour citer cet article :}

Verstraeten, A. (2011). Une brèche dans la culture, d'un bord à l'autre. Témoigner et recevoir le témoignage de la disparition forcée. Alterstice, 1(2), 69-78. 


\section{Introduction}

C'est auprès des « Mères de la Place de Mai » argentines que j'ai appréhendé le témoignage de l'horreur.

Le 24 mars 1976 au petit matin, alors que le pays subit depuis plusieurs années déjà les ravages d'escadrons de la mort parrainés par le pouvoir, l'Argentine se réveille aux mains des militaires. La Junte annonce une " réorganisation nationale » pour " nettoyer » la société de tous les " subversifs apatrides et judéo-communistes » et pour la rendre plus "moderne et occidentale ». Le coup d'État s'inscrit ainsi dans une sombre filiation et veut prendre la suite du Chili de Pinochet. Avec davantage de "classe ». Dans le silence. II ne s'agit pas de passer pour des brutes en exécutant les opposants dans des stades. En revanche, ceux-ci vont subir une vague de disparitions forcées d'une violence rare. Aujourd'hui, les associations argentines de défense des droits humains ${ }^{1}$, avec pour noyau dur les "mères » et les "grands-mères " de la Place de Mai, estiment qu'en sept ans, la dictature a fait trente mille disparus, dix mille morts dans les affrontements, un million d'exilés, que les militaires et leurs proches se sont appropriés cinq cents enfants en bas-âge comme butin de guerre et qu'ils ont utilisé pour cela des centaines de camps de torture et de disparition, des milliers de tombes anonymes, des dizaines de fosses communes.

En 1976, nous n'avions idée ni du sens, ni de l'ampleur de la catastrophe, me disent souvent les « Mères de la Place de Mai ». Un jour, nos enfants ont disparu. Et peu à peu, nous avons su.

La vie de ces femmes se transforme alors en un combat permanent contre la rupture de tous les tabous, contre l'indicible, l'invisible, le mutant, le fantasmatique, l'évanescent. Elles découvrent que les disparus sont enlevés par des escadrons qui ravagent tout sur leur passage, emmenant comme butin de guerre les meubles, la vaisselle, les bijoux, les tableaux, les chiens et même, sommet de l'horreur, les bébés. Aveuglés par des cagoules, ceux qui désormais « n'existent plus » pour la bureaucratie sont jetés dans des Ford Falcon sans plaques d'immatriculation. Ils sont emmenés dans des camps de torture et d'extermination. Ils y perdent leurs noms, remplacés par une lettre et un numéro. Ils sont torturés psychiquement et physiquement, principalement à l'électricité. Puis ils sont, dans leur immense majorité, exécutés. Certains sont endormis par une piqûre de penthotal et jetés vivants à la mer depuis des avions, dans ce que l'on a appelé les «vols de la mort ". D'autres sont fusillés dans des simulacres d'affrontements, et enterrés anonymement dans des tombes anonymes ou dans des fosses communes. L'époque est, me dit Julia ${ }^{2}$, " dantesque » :

Hubo un período donde iban cayendo uno tras otro los compañeros de Gabriel, los amigos, los amigos de mis hijos y... los hijos de mis amigos.

[II y a eu une période où tombaient les uns après les autres les camarades de Gabriel, des amis, des amis de mes fils, et... des fils de mes amis.]

Les familles, qui arpentent les labyrinthes administratifs en tous sens, butent contre un mur de négation et d'impunité. Elles doivent, dès l'entrée, affronter les angoissantes oscillations du langage. Le pouvoir use d'une véritable langue disparitionniste performative de l'effacement de l'autre. II invente peu à peu sa propre terminologie, où les « subversifs » sont " aspirés » dans des "suçoirs ». Les camps sont appelés "club de sport », " trou » ou " aquarium ». Les bourreaux s'appellent "l'ange blond de la mort ", " le Japonais » ou « Petit oiseau ». Commissariats, palais de justice, cabinets d'avocats, tous découragent les demandes d'Habeas Corpus : les disparus n'existent pas. Diego est "sûrement parti avec une blonde ", Ana est " certainement partie rejoindre l'ETA en Espagne ", et leurs mères sont de bien mauvaises mères si elles ne savent pas où diable sont leurs enfants...

Non seulement les disparus sont rayés de la carte sociale, mais leurs proches sont privés de toute attache culturelle, de toute possibilité de dialogue ou de médiation, de tout rituel, de tout langage... De tout langage ? Pas

\footnotetext{
${ }^{1}$ L'usage de cette expression est expliqué dans le texte introductif au numéro (Saillant, 2011)

2 Julia Braun, psychanalyste proche des "Mères de la Place de Mai », est la mère de Gabriel Dunayevich, enlevé en pleine rue par un escadron, à l'âge de 18 ans, le 29 mai 1976, et dont le parcours de "disparu » est repris plus largement dans la suite de cet article. Auteur de divers articles sur le rapport de la psychanalyse à cette terreur, Julia m'a reçue chez elle où j'ai pu enregistrer son témoignage au dictaphone, en 2006.
} 
tout à fait. Contre toute attente, les « Mères de la Place de Mai » se regroupent et à travers leur existence s'amorce une remontée culturelle, depuis le gouffre d'une indicible et isolante horreur vers une forme de pensée partagée malgré tout.

Contre toute attente des militaires donc, et malgré une douleur viscérale, en 1977 des mères de disparus décident de se regrouper. Elles agissent ainsi, à la naissance de leur mouvement, contre l'isolement et la déliaison. Le témoignage, expression d'une intimité, se partage alors avec d'autres (sur)vivants. II participe d'une expression chorale d'une histoire collective qui a touché chacun des acteurs dans son intimité. Le témoignage est mis en réseau. Le témoignage est un partage. En tant que tel, il apparaît comme une réinitialisation du lien social.

Ces mêmes femmes, après s'être regroupées, montent au front en s'exposant sur la Place de Mai. Elles se font visibles devant le siège du gouvernement. Résistance à l'invisible disparitionniste: elles sont là. Si des mères réclament leurs enfants, c'est que ces enfants existent. En désamorçant partiellement le déni, elles forcent un premier pas vers la reconnaissance des crimes. Depuis trente-quatre ans, tous les jeudis, entre quinze heures et quinze heures trente, elles tournent en rond sur la place de Mai.

C'est donc un jeudi de l'année 2003 que je suis allée à leur rencontre. Moi qui me demandais comment j'allais oser évoquer la disparition, moi qui craignais de faire violence à ces femmes en leur demandant de faire ressurgir I'horreur, j'ai été saisie par un flot de paroles. Inutile - et indécent - d'envisager le moindre questionnaire. Pendant qu'elles me parlaient, ces vieilles dames m'embrassaient, me tenaient, me caressaient, m'encourageaient à aller au bout de mon travail. Il était immédiatement devenu vital - et inévitable - d'entrer dans une observation participante, de ne pas nous abandonner mutuellement, de faire un petit bout de chemin ensemble. "Mères ", grands-mères pour moi, elles m'aidaient à les écouter, elles me guidaient dans leurs témoignages qui surgissaient par à-coups puis revenaient en boucle. Elles vomissaient leur douleur à travers les mots et en même temps, tentaient de me les nettoyer, de ne pas m'incommoder. Elles répétaient, écrivaient, mimaient, tentant par tous les moyens d'établir des passerelles d'une rive à l'autre de la brèche. Brèche ouverte dans le sens, ouverte dans notre créance en le réel et en le social, ouverte dans notre espérance en la culture.

Je voudrais défendre ici l'idée que le témoignage de l'horreur est toujours interculturel : il demande une traduction, une médiation des imaginaires, une inflexion du témoin vers un langage toujours réifié et une inflexion de celui qui l'écoute vers l'impensable et l'indicible (donc vers une parole anguleuse, pleine d'aspérités, de vides et d'arêtes tranchantes). II nécessite une mise en lien de la civilisation et de la " décivilisation ", pour reprendre l'expression de Germaine Tillion (1988). Dire - toujours partiellement - la disparition, c'est faire venir la mort de la culture à la bouche du témoin et à l'oreille de celui qui l'écoute.

Je me pencherai donc d'abord sur l'acte de témoigner de la disparition, avant d'en venir à l'acte d'écouter ces témoignages entamés de vides.

\section{Témoigner (malgré tout, malgré soi)}

La violence subie, si elle résulte d'une matrice commune de la terreur, présente tant de déclinaisons, d'exceptions, d'arbitraire, qu'elle isole profondément les victimes. Elle les isole même les unes des autres. Témoigner de sa souffrance, c'est risquer de réactualiser la violence subie, c'est risquer de rajouter chez l'autre victime une nouvelle strate de peurs et de fantasmes qu'elle n'avait jusqu'alors pas imaginés, c'est faire naître cette violence dans l'esprit de "l'étranger » qui l'écoute. Doit-on tout dire au nom de la " vérité " ? La crudité du récit, l'exposition des faits, est d'une violence inouïe. Mais l'atténuation des faits ou leur arrangement, eux, sont une forme de violence feutrée qui, à terme, n'en est pas moins destructrice. Et le témoin semble condamné, s'il souhaite parler, à choisir une forme de violence et à l'assumer. Sinon, il plonge dans le silence complet, participant alors à la perpétuation de la violence telle qu'elle a été pensée par les bourreaux.

Témoigner de la disparition, c'est composer avec la violence au nom d'une utopie. C'est témoigner de crimes invisibles et impunis pour les rendre visibles, pour les porter devant la justice et devant l'Histoire. C'est lutter contre l'isolement physique, moral et social. Si les témoins témoignent, c'est entourés de leur réseau associatif et 
de leur slogan : «memoria, verdad y justicia » (« mémoire, vérité et justice »). Par l’invocation de la mémoire, les familles semblent témoigner pour leurs morts, pour les autres victimes. Par la " vérité », elles semblent s'adresser aux historiens. Par la "justice », elles interpellent leurs concitoyens. Ces trois idéaux se rejoignent dans la nécessité de créer des ponts, des interactions, des confrontations, pour donner un sens aux événements. Or c'est presque impossible. Il semble impossible de donner vraiment du sens à cette chose, entame mentale et morale qu'est l'horreur. Il semble également impossible de créer des ponts, de susciter solidarité ou confrontation, lorsque l'on peine à trouver des interlocuteurs. Mais nous y reviendrons.

La parole (comme "langage incarné ", comme acte d'un sujet s'adressant à un autre sujet, comme expression des émotions et expression d'un point de vue sur le monde...) semble impossible si le langage qu'elle utilise comme outil est piégé. Les militaires ont déjoué, détourné, renversé la fonction première du langage : exprimer ce qui est. Ils ont utilisé les mots pour occulter ce qui est et faire apparaître ce qui n'est pas. Ils ont bombardé la société de mots creux et de mensonges, et quelque chose reste de cette falsification permanente. Les témoignages ont lieu malgré tout, mais transis d'impuissance, empreints d'ambivalence et de contradictions. S'ils ont lieu, c'est en interrogeant dans le même temps les rapports entre langage, vérité et représentation, entre dicible et indicible, humain et inhumain. Lors de nos entretiens, les témoins n'ont de cesse de souligner la difficulté de " traduction » de leurs pensées en des termes «interculturels »: des termes qui me permettraient de les appréhender. Nombreux sont ceux qui font appel à la Shoah, implicite culturel de l'horreur, matrice mentale des indicibles. Ils semblent utiliser un langage partiel ou temporaire. Ils affirment le caractère exploratoire et " entre-deux » de leur parole. Haydée, mère de disparu ${ }^{3}$, tente par exemple de faire passer ses impressions en même temps qu'elle désosse les mots:

No hay nada que te lleve más miedo y más angustia que la desaparición. Porque si te... si a un hijo tuyo, te lo matan, a vos te entregan un cadáver. Es muy doloroso. La muerte de un hijo no te la compensa nada. Pero nuestro hueco negro, esto es la desaparición. Es un hueco negro que siempre te acompaña la vida. Es eso [muestra el vacio alrededor suyo con un amplio movimiento de los brazos], "desaparición". Y te impone muchisimo más miedo. Prostraron un país en el miedo! Es una cosa muy retorcida, muy perversa y muy ideada. [...] Ni una huella, nada. Es... es horrible. Es algo que te dejó un vacío... negro... un... al... algo que la cabeza no... y que el corazón no... no... Una cosa permanente. Nada, nada. Hoy tenés tu hijo, mañana no sabrás absolutamente nada más de él. [...] Yo lo vivo como un hueco negro enorme. Una ausencia... indefinida y... tremenda.

[II n'y a rien qui te donne plus de peur et plus d'angoisse que la disparition. Parce que si tu... si un de tes fils est tué, on te rendra un cadavre. C'est très douloureux. La mort d'un enfant, rien ne la compense. Mais notre trou noir, ça, c'est la disparition. C'est un trou noir qui accompagne toute ta vie. C'est ça [elle montre le vide autour d'elle dans un ample mouvement des bras], " disparition ». Et ça t'impose immensément plus de peur. Ils ont prostré un pays dans la peur ! C'est quelque chose de très tordu, très pensé, très conceptualisé. [...] Ni une trace ni rien. C'est... c'est horrible. C'est quelque chose qui t'a laissé un vide... noir... un... quel... quelque chose que la tête ne peut... et que le cœur ne... ne... une chose permanente. Le néant, le néant. Aujourd'hui tu as ton fils avec toi, demain tu ne sauras absolument plus rien de lui. [...] Je le vis comme un trou noir énorme. Une absence... indéfinie et... terrible.]

La résistance naît dans le témoignage, puis le témoignage soutient, renforce, alimente la résistance. II contre la mort du langage et du symbolique par tâtonnement verbal et gestuel, et par des inventions (banderoles, silhouettes en carton, bougies, photos, vêtements des disparus, foulard blanc porté sur la tête pour rappeler le lange de l'enfant disparu); il contre le silence par la parole travaillée et par les chansons, mais aussi par les cris, les pleurs et les hurlements des manifestations; il contre l'impunité en attirant ainsi les regards, puis les médias et, enfin, la justice; il contre la massification et la déshumanisation en individualisant les victimes, en incarnant les

3 Haydée Gastelú de García Buela est l'une des toutes premières femmes à s'être regroupées pour lutter contre le silence, elle est l'une des fondatrices du mouvement des « Mères de la Place de Mai » et mère de disparu. Son fils, Horacio Oscar García Gastelú (21 ans), a " disparu » le 7 août 1976 avec sa petite amie Ada Victoria Porta (" Dodo », 18 ans). Horacio étudiait la biologie, était membre des JP (Jeunesses péronistes) et de la UES (Union des étudiants du secondaire). II était conscrit dans l'infanterie de la Marine au moment de l'enlèvement. Ses supérieurs, qui se sont toujours refusé à faire des recherches le concernant, l'ont alors cyniquement déclaré « déserteur ". Le 24 avril 2001, les anthropologues légistes ont annoncé à Haydée et à son mari Oscar qu'ils avaient retrouvé le corps d'Horacio parmi les victimes du Massacre de Fátima (Province de Buenos Aires, 20 août 1976). Son parcours de détention a pu être retracé. Nous savons désormais qu'il était séquestré dans le sous-sol du siège des services secrets à Buenos Aires. II a été enterré le 25 août 2001. Ces propos ont été recueillis au dictaphone, en 2004. 
détails de chaque vie et de chaque mort. Enfin, il contre la déliaison par les mises en liens de ces histoires incarnées, et par les mises en liens des témoins.

Témoigner est un acte de résistance. On s'y attelle avec un certain sentiment d'illégitimité, on s'y attelle pour les autres : nombreux sont les témoins qui estiment que les véritables victimes (et par-là, les véritables témoins) sont mortes. Ils surmontent l'inhibition. Ils se font violence pour témoigner. Beatriz, mère de disparu ${ }^{4}$, s'est fourré des serviettes de bain dans la bouche tous les soirs pendant trente ans, pour pouvoir hurler sa douleur en silence, sans pouvoir raconter. Un jour, elle a franchi la porte de l'association des mères et depuis, dit-elle, elle " apprend » à parler. Ceux qui parlent beaucoup, comme Primo Levi après la Shoah, peuvent finir par se suicider. Dire est impossible. Se taire est impossible aussi. Cela, Jorge Semprun et Elie Wiesel (1995) l'ont déjà souligné. Les témoins se trouvent pris au piège du témoignage. C'est une exigence à laquelle ils ne peuvent échapper. II leur est impossible, psychiquement, moralement, de ne pas témoigner. Mais dans le même temps, témoigner est impossible ! Un témoignage "plein », entier, entièrement plein de "vérité » est utopique. Il est toujours fait de creux, de vides, d'aspérités, de «trous noirs ».

Ceux qui survivent ne portent pas seulement en eux les souvenirs des sévices qu'ils ont dû personnellement endurer, ils sont porteurs des souffrances et des exécutions de ceux qui ne sont jamais " réapparus ». Porteurs du stigmate de la disparition, les témoins sont à la fois le citoyen, l’humain qui aujourd’hui témoigne... et l'être réduit à l'état de corps qui, resté dans le camp, hante le récit d'un espace et d'une temporalité parallèles, hante la recherche de la "vérité » d’une violence qui lui est indissociable. Selon Annette Wieviorka (1998, p.61), «le survivant est en fait tout à la fois mort et vivant, personnalité clivée qui, tout en continuant son chemin est accompagné par son double mort comme par son ombre ». Le survivant de la disparition semble accompagné, au présent, d'un lui-même au passé, toujours disparu. "Une partie de moi est morte là-bas ", entend-on souvent. L'enlèvement, la séquestration, la torture physique et psychique sont si profondément ancrées dans la mémoire qu'ils semblent continuer d'exister au présent, malgré une vie quotidienne de survivant qui par ailleurs s'écoule. Dans leurs cauchemars ou à la suite de multiples étincelles (les ustensiles d'un dermatologue sur la peau pour Laura, un chant d'oiseau pour Victor, une musique à la radio pour Carmen... ), les survivants ${ }^{5}$ se trouvent plongés à nouveau dans le passé toujours potentiellement présent. Les proches de disparus, qui eux ont vécu une angoisse démesurée, innommable, sont également clivés : ils sont porteurs de leurs disparus, et ils sont eux-mêmes, malgré leur vie au présent, toujours partiellement enferrés dans leur gouffre d'angoisse, ils sont toujours partiellement soumis à la terreur. Le temps a continué sa route, mais il s'agit d'une avancée paradoxale. Les témoins sont des vivants, des acteurs sociaux paradoxaux.

Ces témoins sont des personnalités clivées en ce qu'ils sont des acteurs sociaux porteurs de la mort du social, de "l'envers» de la culture. Ils sont donc, pour nous qui les recevons depuis "l'endroit » du décor, éminemment déstabilisants.

\footnotetext{
${ }^{4}$ Le fils de Beatriz, Jorge Marcelo Dyszel, et sa bru, Mirta Schwalb de Dyszel, âgés respectivement de 21 ans et 20 ans, ont disparu le 18 mai 1978. Ils étaient mariés depuis dix mois. Beatriz n'a plus jamais eu de nouvelles d'aucun des deux. Les familles de Jorge Marcelo et de Mirta ont commencé par déposer une déclaration auprès de la Ligue argentine des droits de l'homme, puis ont déposé des Habeas corpus (toujours rejetés), ont approché la presse (toujours réticente et menacée), ont sollicité les rabbins, ont voyagé à Londres auprès d'Amnesty International (où ils ont pris la mesure de l'ampleur de la disparition), au Vatican pour voir le Pape (qui ne les a pas reçus)... Si Beatriz a, dès le départ, participé aux manifestations, elle n’a intégré l'association des « Mères de la Place de Mai - ligne fondatrice » qu'une fois retraitée, en 2004. Divorcée, elle devait travailler pour vivre, et s'exposer sur la Place de Mai c'était risquer le renvoi, les employeurs craignant toujours une contagion de la disparition. C'est au siège de l'association, autour des albums de souvenirs des disparus qu'elle constituait, que j'ai recueilli son témoignage au dictaphone, en 2006.

${ }^{5}$ Laura Seoane et Victor Basterra ont survécu respectivement à deux semaines et à trois ans et demi de séquestration à L'ESMA, immense camp de torture et de disparition dans les locaux de la Marine, à Buenos Aires. Carmen Aguiar de Lapacó a survécu au camp «EL Atletico » (le " club de sport »), dans le sous-sol d'un bâtiment de la police, à Buenos Aires. Elle y a perdu de vue sa fille, Alejandra Lapacó, qui avait 19 ans et étudiait l'anthropologie. Alejandra et son petit ami Marcelo Butti Arana sont aujourd'hui encore et toujours disparus. Les témoignages de Laura, Victor et Carmen ont été filmés dans le cadre des repérages puis, pour les deux premiers, du tournage de notre film documentaire Victor (co-réalisé avec Cécile Verstraeten, produit par Dérives productions, Luc et Jean-Pierre Dardenne, Liège, Belgique, 2009, 48 min.)
} 
Ce témoignage de l'horreur est alors comparable à une situation interculturelle, tendue mais créative, incertaine, faite d'efforts mutuels et d'inflexions. La rencontre entre les (sur)vivants et le " reste » de la société (" reste » qui n'a pas été directement confronté à la violence décivilisatrice) ne peut se faire qu'à travers le témoignage. Et elle ne peut se faire qu'à travers un témoignage précis. Elle suppose que les témoins comme leurs interlocuteurs refusent la déshumanisation et la massification, refusent que des humains, devenus des numéros puis des «paquets» soient engloutis dans les vagues du Rio de la Plata ou emmêlés dans des fosses communes. La rencontre n'est possible que si l'on refuse de s'arrêter, sidérés, au seuil de l'idée qu'il ait pu y avoir " trente mille disparus ». Ce chiffre, en un sens, prolonge la massification et, sinon la déshumanisation, au moins la déréalisation des victimes. Pour que, anthropologue, je ne perde pas le lien sensible avec mes interlocuteurs et pour que je puisse construire avec eux une relation sensée; pour que vous, lecteurs, puissiez aborder la disparition forcée non comme une horreur théorique, mais comme une horreur touchant à notre commune humanité... j'en reviens, comme les "Mères de la Place de Mai » elles-mêmes, à la nécessité de retranscrire et de répéter encore des histoires de vies. Litanie ? Lien de vie. Entame de la pensée ? Entame de cet article.

Gabriel Dunayevich, par exemple, a disparu à l'âge de 18 ans, le 29 mai 1976. Élève au collège national de Buenos Aires, il militait au sein de l'Union des étudiants du secondaire. II a été enlevé en pleine rue avec une camarade, sous les yeux de deux autres amis. Julia, sa mère, n'obtient plus aucune nouvelle avant 1985. C'est un juge qui l'appelle alors. Il peut affirmer que Gabriel est mort assassiné, que son corps a été abandonné dans les rues dans un simulacre d'affrontement puis enterré anonymement. À l'époque où son corps avait été abandonné dans la rue, Gabriel aurait pu être identifié. Les autorités avaient fait une autopsie et pris ses empreintes digitales, mais ne les avaient pas comparées à leurs fichiers. Absurde. Arbitraire. Des photographies des cadavres avaient été prises, mais personne n'avait été convié à les reconnaître. Déliaison. Ce juge, qui enquête en réalité sur le Massacre de Fátima, vient de recevoir un témoin et, sur un coup de tête, de lui montrer aussi ces photos. Or, le témoin identifie Gabriel. Julia et son mari l'identifieront aussi. Ils découvrent dans le dossier du tribunal que le corps de Gabriel a été trouvé le 3 juillet, à l'aube, en banlieue de Buenos Aires, aux côtés de deux autres corps. Les corps gisaient dans une mise en scène macabre, avec des pancartes sur lesquelles était écrit un mensonge : "J'étais montonero ${ }^{6}$ ". Les autopsies avaient révélé une exécution à la mitraillette. Julia et son mari se rendent dans le quartier et discutent avec les voisins, qui se souviennent très bien de la fusillade. C'est donc grâce à divers témoignages que peu à peu, I'horreur indistincte de la perte du fils prend des contours plus précis. S'ils ne sont pas moins horribles, au moins ces contours permettent-ils d'élaborer du sens :

Lo importante es que tuvimos testigos de... del secuestro, y también tuvimos testigos de la muerte. Y pudimos saber que entre el secuestro y la muerte no pasó tanto tiempo, del 29 de mayo al 3 de julio. Poco tiempo es el alivio de que el sufrimiento no fue... lo que en esos momentos pensabamos que... había sido muy largo.

[Ce qui est important c'est que nous avons eu les témoins de ... de l'enlèvement, et les témoins de la mort. Et nous avons pu savoir qu'entre l'enlèvement et la mort, il ne s'est pas passé tant de temps, du 29 mai au 3 juillet. L'idée que cela ait duré peu de temps nous soulage en ce que la souffrance n'a pas été... telle que ce que, à ce moment-là, nous imaginions. Cela n’avait pas été très long.]

Les informations fournies dans le dossier sont toujours contradictoires, et il en va de même concernant le lieu d'ensevelissement anonyme de Gabriel et de la jeune femme qui se trouve avec lui. Julia et son mari mettent donc un moment avant de retrouver les lieux. Quand les anthropologues légistes peuvent enfin se pencher sur les ossements récupérés, ils ont du mal à identifier formellement Gabriel. Car ils ne disposent pas encore de la technologie des analyses ADN. Alors qu'ils sont près du but, un juge leur retire l'affaire, et les ossements sont envoyés à un ossuaire commun. Julia ne pourra plus récupérer le corps de son fils. Retour de l'arbitraire et de l'impunité qui ont déjà broyé tant d'intimités :

Y esta la enorme decepción, otro momento tremendo, otra vez de pensar que no vamos a poder tener por lo menos el cuerpo, los restos, que una vez más se perdió... por la actitud de un juez.

[La déception est énorme, c'est un autre moment terrible, de penser que nous n’allons pas pouvoir récupérer le corps, les restes, que ça a été perdu une fois de plus... à cause de l'attitude d’un juge.]

\footnotetext{
${ }^{6}$ Montonero : militant dans la branche armée du péronisme de gauche.
} 
Quelque temps plus tard, Julia reçoit une lettre d'une Argentine exilée au Mexique. Survivante de "El Vesubio » ("Le Vésuve »), elle y a vu Gabriel. Ils étaient tous les deux dans le sous-sol, l'un à côté de l'autre, enchaînés au sol... Cette femme raconte que Gabriel a dû subir les tortures « spéciales » que les bourreaux réservaient aux juifs (« juif, juif de merde »), en imitant les nazis. Cette femme raconte que les détenus s'étaient promis que celui qui survivrait irait dire aux familles ce qu'il était advenu de tous les autres. Ils mémorisaient tous les noms et gravaient dans leurs têtes un maximum d'information. Elle honorait sa promesse en contactant Julia.

Témoigner, à chaque maillon de la chaîne, c'est faire renaître du social et du culturel. À travers liaisons et resymbolisations, c'est faire émerger une " culture malgré la mort », une " culture avec l'horreur »... contre la mort de la culture et contre l'horreur qui jaillit du gouffre.

\section{Recevoir le témoignage}

J'ai eu besoin des "Mères de la Place de Mai » pour comprendre le crime contre l'intimité et contre l'humanité (autant que possible, en ayant conscience que c'est fondamentalement impossible). Parce qu'elles ont accompagné mon appréhension de l'horreur, parce qu'elles ont façonné mon rapport à l'indicible et à l'irreprésentable, j'ai osé me tourner ensuite vers des survivants des camps. J'ai mis du temps à aller à leur rencontre parce que je voulais une place juste, je voulais éviter le sensationnalisme et j'avais aussi, c'est vrai, un mouvement de recul face au spectre de la torture. Je n'étais absolument pas sûre d'en supporter l'évocation.

Cette nouvelle étape de mes recherches me conduit à interroger le cheminement nécessaire non aux témoins, mais à ceux qui les écoutent, pour que le témoignage soit rendu possible. Et pour comprendre ce cheminement, peutêtre faut-il commencer par se pencher sur ceux qui le refusent, et qui sont la majorité : le témoignage est empêché par le bourreau, bien sûr, mais aussi par la foule de ceux qui ne veulent pas voir ou qui veulent rester « neutres » et " garder une certaine distance » avec la fureur de l'Histoire.

Soulignons donc que le témoin de la disparition est toujours une victime de la disparition : les bourreaux, eux, ne parlent pas et définissent par là-même une part de leur envergure criminelle. Dans chacun de leurs actes de terreur, par l'effacement des noms et des traces, par le brouillage des pistes, par les menaces à l'encontre des proches ("si vous approchez des associations de défense des droits humains, vous ne reverrez pas votre fils ») ou des survivants (« si tu parles, on te retrouvera »), les bourreaux ont voulu empêcher le futur témoignage.

Une fois la parole des victimes affirmée malgré eux, les bourreaux empêchent qu'elle n'aboutisse pleinement : tout témoignage a un but, et celui des "Mères de la Place de Mai », " mémoire, vérité et justice », est inatteignable. Les bourreaux, acteurs majeurs de la disparition, occultent leur part de la mémoire et de la vérité historique. Et rejettent bien sûr la justice. Tant qu'ils refusent de reconnaître leurs crimes et de livrer leur part de témoignage, le dialogue est impossible et toute tentative de reconstruction du sens est partiellement avortée d'entrée.

Les « neutres » quant à eux, ceux qui disent qu'ils « ne savaient pas » ou qu' « il ne faut pas rouvrir les blessures du passé ", ceux qui prônent la "réconciliation " au nom de "l'unité nationale ", ne mesurent pas l'ampleur de la catastrophe sociale advenue. Ils refusent de prendre en compte la "décivilisation » comme si elle menaçait d'effondrement la "civilisation ". Ils ne peuvent envisager que la destruction du social puisse jaillir du social luimême et que le social puisse encore, cahin-caha, tenir debout. Or, sur la scène sociale, le témoin surgit comme un signe vivant de l'envers du décor. Il est la part visible de l'invisible. C'est lui qui, en un sens, fait advenir la disparition dans les consciences. Car si la disparition est produite par les bourreaux, ceux qui la font remonter à la surface sont les témoins. Ce sont donc eux, dans un premier temps, qui horrifient. Ce sont eux qui sont perçus comme sources de la terreur. Haydée raconte ainsi combien il est difficile de faire affleurer cette réalité, combien ses interlocuteurs ont pu vouloir se "protéger » en rejetant les témoins dans les sphères de la folie. Combien ses interlocuteurs ont pu vouloir ne pas voir et ne pas savoir :

Sabíamos perfectamente que existían campos de concentración, porque volvian algunos. Sabíamos... [Habla suspirando :] que los torturaban, sabíamos que los tiraban al Río... Pero no podíamos creerlo ! Fuimos sabiendo, de a poco. Pero si lo contabamos, veinticinco años atras, nadie nos creía! Cuanto más horrible es lo que vos puedes contar, lo menos te cree la gente. Y lo menos quiere escucharte. 
[On savait parfaitement qu'il y avait des camps de concentration, parce que quelques-uns en revenaient. On savait... [Elle parle dans un soupir :] qu'ils les torturaient, on savait qu'ils les jetaient au fleuve... Mais on ne pouvait pas le croire. On a su, petit à petit. Mais si on le racontait, il y a vingt-cinq ans, personne ne nous croyait! Plus ce que tu as à raconter est horrible, moins les gens te croient. Et moins ils veulent t'écouter.]

Par l’horreur qu'il charrie, le témoin est, dans un premier temps, inaudible.

Par le danger qu'il signifie, le témoin, surtout, fait peur. Haydée souligne qu'aux débuts de la ronde sur la Place de Mai, lorsqu'elle portait son foulard blanc sur sa tête et qu'elle était ainsi "stigmate " (Goffman, 1975) de la disparition, elle ne saluait personne. Pas même un cousin qui passait dans la rue. "II ne fallait pas le marquer », disait-elle. Car le seul contact avec une personne elle-même en lien avec la disparition apparaissait comme un risque de disparaître à son tour. On ne savait pas très bien où s'arrêtait la disparition. Le sentiment d'arbitraire qui se dégageait de la nébuleuse répressive déliait ainsi le social, ses membres étant toujours plus tentés de se rapprocher d'un noyau dur de "normalité ", toujours tentés de s'éloigner un maximum de marges dont on ne saisissait pas les tenants et aboutissants.

Cette violence dont les témoins sont à la fois cibles et porteurs les pousse aux marges impensées du social. Les témoins sont un insupportable lien avec l'envers du décor, avec la "dé-civilisation ». Cette mise en lien fait violence à toute idée de neutralité. Elle nous rappelle que toute société est "grise » : ni noire, ni blanche, mais "grise " au sens d’une "zone grise » d'enchevêtrement du bien et du mal telle que Primo Levi (1989) a pu la définir.

Le témoignage, en somme, semble rendu impossible par le spectre de l'indicible et de l'irreprésentable. L'indicible est un piège de la pensée. Catherine Coquio (1999, p.61) nous met en garde contre la piété (et l'idée que ce que l'on ne peut dire, il faut le vénérer) puis contre le cynisme (et l'idée que ce que l'on ne peut dire, il ne faut plus y penser). En n'utilisant l'indicible ni comme bouclier ni comme absolu, il s'agit d'admettre qu'un trou noir aspire le sens et les sens.

II s'agit de comprendre que la disparition reste partiellement évanescente. On ne peut jamais faire que l'approcher. On ne peut la toucher, on ne peut la penser autrement qu'en tant que silhouette, enveloppe contenant du vide : on ne peut la penser qu'en tant que contour de l'impensable, moulage de l'infigurable. Chaque fois que l'on tente de l'exprimer, ce qui affleure peut-être le plus... c'est l'impossibilité à exprimer. Et l'impossibilité à dire trouve son prolongement dans d'autres formes que celles du récit. L'indicible est aussi une sensation physique. Une sensation d'asphyxie, d'oppression, d'urgence. Il empoisonne, enrage, exaspère, et pire encore. Les mères de disparus et les survivants en témoignent : béance, trou, nœud, stupeur, terreur, colère, angoisse, sont des termes qui peuplent leurs témoignages. Tout cela constitue l'indicible.

La disparition se définit par des impossibilités. Elle se définit par le fait d'être une désorganisation. On en " reçoit " néanmoins quelque chose comme on se brûle, comme on se blesse. On est « heurté » par la disparition.

Écouter un témoignage de la disparition, c'est donc n'être ni bourreau, ni neutre. C'est être ébranlé. À partir de la commotion émerge une empathie pour les témoins. C'est à partir de cette empathie qu'un partage de sens (même minime) est possible. Et c'est à partir de ce partage de sens que (re)naît la possibilité d'une culture commune, d'une société viable.

Pour qu'il puisse y avoir "passage du témoin » entre ceux qui ont fait l'expérience de la violence extrême " décivilisatrice » et ceux qui les écoutent, la parole doit bien sûr être accompagnée d'une relation. En ce sens, le témoignage est une figure paradigmatique du lien social (Ion et Peroni, 1995). Un contrat implicite de solidarité le sous-tend, comme condition de possibilité. Ce contrat de solidarité veut que le récit de vie soit une co-construction d'un sens provisoire et tâtonnant impliquant celui qui l'énonce, celui qui le transmet, celui qui l'écoute.

II m’a fallu me rendre à l'évidence : se positionner en « lien » entre des humains qui témoignent et d'autres qui les écouteront ou les liront, c'est être en permanence sur le fil. À la racine de ce travail se trouvent la révolte et l'incompréhension face à un crime innommable. Lorsque « ma » première " Mère de la Place de Mai » m’a prise 
dans les bras après une ronde, est née une empathie viscérale. Parfois, la " décivilisation " nous emporte, mes interlocuteurs et moi-même, dans un gouffre de pessimisme. Mais la capacité de résistance des victimes, la force des liens qui unissent les témoins et ceux qui les écoutent, la chaleur du corps à corps des manifestations, l'intensité d'un regard après un récit partagé... nous empêchent d'abandonner la voie sur laquelle nous nous sommes engagés. Je ne laisse pas tomber parce que je me sens liée par un contrat implicite de solidarité avec d'autres êtres humains, parce que j'ai de plus en plus le sentiment de leur devoir d'aller jusqu'au bout. Ce sont les témoins, dans ce qu'ils me racontent, lorsqu'ils "réveillent mes morts " par exemple, qui suscitent mes ras-le-bol. Mais ce sont eux encore qui me ramènent à mon travail, parce que je les aime, parce qu'ils $m^{\prime}$ ont appris à aimer notre commune humanité malgré tout, malgré le cheval de Troie qui trône désormais dans mon esprit.

Lorsque l'on est "heurté " par la disparition, alors seulement l'impossibilité à dire nous pousse à vouloir écrire, l'impossibilité à représenter nous pousse, par exemple, à vouloir filmer et l'impossibilité à trouver la « vérité » nous pousse à l'exiger. Moteurs paradoxaux. Ainsi que le souligne Luba Jurgenson (2003, p. 371), « la parole est motivée par cela même qui empêche l'expérience concentrationnaire d'affluer complètement à la surface; ce reste, ce sédiment qui l'alourdit et en fait l'objet d'un désenfouissement infini, est précisément ce qui exige, en premier lieu, d'être dit ». Ce sont les traces (les restes, les empreintes, les sédiments) de la disparition qui nous ramènent sans cesse à elle et nous poussent à ne pas baisser les bras. Si nous ne pouvons assimiler le non-sens, nous pouvons en revanche lutter contre cette entame et nous accrocher à un reste de pensée. Abdiquer face au déni, face à l'indicible et à l'irreprésentable serait annuler complètement la pensée, annuler nos regards intimes sur le monde, annuler toute possibilité de mise en lien de ces regards et donc, annuler le social et la culture.

La mise en lien des témoins entre eux, puis la construction de traverses vers ceux qui les écoutent (d'un bord à l'autre de la brèche décivilisatrice, de l'envers vers l'endroit du décor et réciproquement) met en branle la pensée. Partant de bribes du réel, partant d'expériences intimes, cette relation exploratoire nous amène à penser l'histoire collective. Elle permet une co-naissance du sens. Le témoignage se loge quelque part en équilibre sur une de ces traverses. II cherche à " penser l'avenir à partir du passé, le collectif à travers le singulier, l'inhumain à travers I'humain, le "mutisme du meurtre" [...] à travers la parole rescapée, le passage à l'acte à travers le travail sur la langue » (Coquio, 1999, p. 60).

En ce sens, témoigner, c'est agir politiquement. C'est lutter pied à pied contre la violence disparitionniste. Dans chacun des leurs actes militants, dans leurs témoignages comme dans leur ronde hebdomadaire et comme dans chacune de leurs manifestations, les « Mères de la Place de Mai » et leur réseau militant ont comme points de mire le passé (les engloutis) et l'avenir (avec la peur d'un retour de la violence et de la terreur). Le témoignage, formulé par des vivants pour ceux qui n'ont pu survivre, est destiné à ceux qui vivront. Il est destiné à laisser une trace, une passerelle vers le "reste " (" reste " d'humanité, reste de la société, générations à venir). Il cherche à rendre possible une forme de partage et de transmission et, par-là même, une forme de société viable.

\section{Transmission et interculturalité}

Ce que je voudrais souligner ici, c'est combien un témoignage de l'horreur disparitionniste, en équilibre sur un fil tendu entre dicible et indicible, est une négociation complexe. Le témoin, porteur d'un billet aller-retour vers la rupture de tous les tabous, rebrousse chemin vers la parole. Il est obligé d'abandonner en chemin une partie de la réalité de la disparition, une partie de la vérité (au sens de l'épaisseur, de la complexité du vécu), s'il veut être compris de ses interlocuteurs "normaux ". Ses interlocuteurs, qui n'ont jamais passé la frontière de la violence extrême, sont obligés d'avancer jusqu'à elle, de se tenir au poste frontière en quelque sorte. Ils sont obligés d'admettre qu'ils ne peuvent comprendre, mais ils sont aussi obligés de repousser un peu plus loin leur tolérance à l'écoute de la violence. Ils sont tenus de tolérer l'impensable et l'indicible comme des brèches ouvertes dans la pensée. Ils sont bien obligés d'admettre la "décivilisation» comme un cheval de Troie dans la civilisation, la rupture de tous les tabous comme une entame de la culture. Ils constatent que, s'ils pensaient partager globalement la même culture que les témoins, ceux-ci parlent en réalité depuis la mort de la culture. Quant aux bourreaux, ils incarneraient davantage la culture de la mort. Le témoignage prend forme (une forme tremblotante, armée d'un langage toujours provisoire, choisi par défaut) entre ces trois "cultures", entre ces trois univers de 
sens (où le bien et le mal, la douleur, la vie et la mort, l'éthique, le langage, le passé et le présent, entre autres... n'ont pas le même sens). Pour qu'il y ait trans-mission, il faut qu'il y ait un parcours trans-culturel.

Le témoignage de la disparition semble devoir être "interculturel » : il se loge dans les petits interstices de lumière qui filtrent de la nébuleuse d'impunité, il émerge de la brèche ouverte dans la culture, il tente de recoller les bribes éparses du lien social.

\section{Références bibliographiques}

Coquio, C. (dir.). (1999). Parler des camps, penser les génocides. Paris : Albin Michel [coll. Idées].

Goffman, E. (1975). Stigmate. Les usages sociaux des handicaps [A. Kihm, trad.]. Paris : Éditions de Minuit [coll. Le sens commun].

Ion, J. et Peroni, M. (coord.). (1995). Engagement public et exposition de la personne. Actes du colloque du CRESAL, Saint-Étienne, 3-5 octobre 1995. La Tour d'Aigues : Éditions de l’Aube.

Jurgenson, L. (2003). L'expérience concentrationnaire est-elle indicible? Paris : Éditions du Rocher.

Levi, P. (1989). Les naufragés et les rescapés, Quarante ans après Auschwitz (A. Maugé, trad.). Paris : Gallimard [coll. Arcades].

Saillant, F. (2011). Droits humains et témoignages : l'épreuve de la culture. Alterstice, 1(2), 3-8.

Semprun, J. et Wiesel, E. (1995). Se taire est impossible. Paris : Éditions Mille et une nuits/Arte Éditions.

Tillion, G. (1988). Ravensbrück. Paris : Seuil [coll. XX siècle].

Wieviorka, A. (1998). L'ère du témoin. Paris : Plon/Hachette Littératures [coll. Pluriel Histoire]. 